\title{
Engage with, don't fear, community labs
}

\author{
Ellen D Jorgensen \& Daniel Grushkin
}

\begin{abstract}
The do-it-yourself biology movement has exploded in recent years, culminating in the formation of the world's first community laboratory, opened late last year. As this grassroots effort continues to grow, professional biomedical researchers stand to benefit from partnering with the legions of garage biotechnology enthusiasts.
\end{abstract}
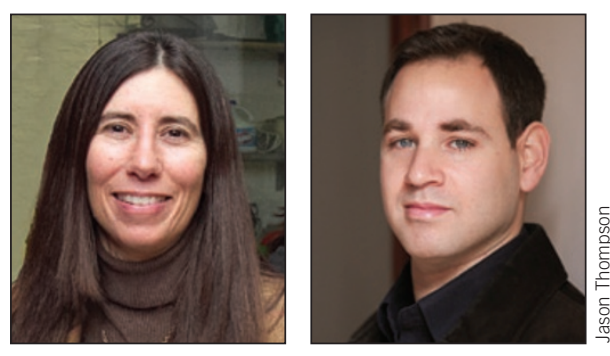

In December 2010, we celebrated the opening of the world's first community biotechnology laboratory by throwing a party in Brooklyn, New York. An eclectic mix of more than 100 guests showed up, including artists, roboticists, furniture designers and financial consultants. Even agents from the US Federal Bureau of Investigation (FBI) popped by. But one group of professionals was conspicuously absent from the party: career scientists. This was unfortunate as the event was an important manifestation of a grassroots movement that has the potential to radically affect biomedical research.

The lab, called Genspace, was the product of two years of hard work by seven people from widely disparate backgrounds. Two of us are biomedical researchers with graduate degrees and over 40 years of combined experience running labs, but the other five are college students or citizen scientists frustrated by the lack of opportunity to conduct self-initiated, hands-on research. Inspired by the potential for crowd-sourced medicine and the promise of synthetic biology, we were eager to get involved, but we had no venue to support our enthusiasm. For the professionals, we yearned for an environment where we could work on pet projects without corporate deadlines or faculty responsibilities. But we also felt that, given the sad state of science literacy in this country, anyone excited about doing science should be encouraged by those with the knowledge and resources to help.

We are not the only group to gather around the idea of do-ityourself biology. Over the past few years, garage biologists have gathered together all over the world, most prominently in Boston and San Francisco (see Nat. Med. 15, 230-231, 2009). However, our lab-located on the seventh floor of a warehouse full of art and design studios off of bustling Flatbush Avenue in downtown Brooklyn-marks a turn in the movement's

\section{Even agents from the FBI} popped by. evolution. Genspace is the first lab modeled on a gym-style membership: for $\$ 100$ per month, individuals with any level of expertise and background can join the lab and become equal participants in our community of biotech enthusiasts. The two caveats are that all projects meet our safety requirements and that members mesh with the group-we are a community after all. As we go forward, other groups will undoubtedly follow suit by developing similar dedicated spaces.

In the past, collectives like ours have been critized as potential havens for bioterrorism by some and as useless endeavors by others. Neither of these statements is true-but getting that message across has proven difficult. Through our work with the FBI, we've gone a long way in quelling biosecurity fears, but our relationship with the biomedical community is still embryonic.

So how does Genspace fit into the larger picture of biomedical research? In the same month that we opened our doors, the US Presidential Commission for the Study of Bioethical Issues released a report on synthetic biology calling for the public, and by extension academic institutions, to embrace citizen scientists. "They demonstrate that science and its oversight do not belong exclusively to experts, highly trained professionals or government officials. Science is a shared resource, affecting and belonging to all citizens," the authors wrote.

In small ways, citizen scientists already contribute to the fields of medical information and genomics, and their participation is rapidly growing. A profusion of websites, including Patientslikeme.com and Cancercommons.org, are making personalized investigation easier. With these, patients fuse the power of social networks with medical research by pooling vast amounts of self-reported data to improve treatments and protocols (see Nat. Med. 16, 953-955, 2010).

Presently, we think it's a mistake to judge the worth of grassroots efforts by asking whether their contributions to biomedicine can directly compete with those of traditional research institutions. The potential exists, but it's too soon to tell. There is always the tantalizing possibility that the fresh viewpoints offered by the diverse participants could lead to new applications for existing technologies-and make real advances in biomedicine some day. We are not yet at the level of, say, synthesizing an antimalarial drug in yeast. But we may not have to be to contribute to public health. One of Genspace's members is designing a portable water quality monitor for disaster areas based on spectrophotometry: an example of how a citizen scientist with a good idea can potentially save lives.

In tandem with innovation, community labs are biomedicine's ideal ambassadors to the public. Genspace's scientific advisory board is made up of researchers and biosafety professionals who have stepped forward to volunteer their expertise because they see our potential to promote greater public awareness and deeper understanding of cuttingedge technologies and the ethical and social issues that they raise. By providing outreach in the form of courses and workshops for nonscientists in subjects such as biotechnology, we make the latest medical advances more accessible, understandable and less threatening. It's harder to fear something one has actually done side by side with one's high-schoolage son or daughter.

For the first time in nearly a decade, Americans perceive the field of biology as deeply relevant to their lives-so relevant, in fact, that they want to contribute. It would be self defeating to the biomedical research community to neglect a grassroots movement fueled by the spirit of inquiry. The community lab idea is an experiment, but an extremely important one. We don't know where it will lead, but even if the sole benefit is to enhance science literacy, that would be enough.

Molecular biologist Ellen D. Jorgensen is an adjunct assistant professor at the New York Medical College in Valhalla and the president of

Genspace. Daniel Grushkin is a science writer in Brooklyn, New York and vice president of Genspace. 